\title{
Some like it hot
}

Working in a Mexican restaurant during his teenage years, Mark Buchanan discovered his love for jalapeños. Since then he has climbed higher and higher on the Scoville scale.

B eautiful, dark-green jalapeño peppers will make your tongue burn wonderful! But as I later discovered, the jalapeño is a mild creature compared to the bird's eye chilli, which gives an aggressive fire to Asian curries and soups. And that heat, I now know, bears no relation to the savage pain produced by a Naga or Bhut jolokia chilli, or the trauma of the pointy-tailed Carolina Reaper, currently the world's hottest pepper. Bred as a hybrid in 2013 by 'Smokin' Ed Currie in South Carolina, the Reaper reaches 2.2 million Scoville units on the scientific scale used to measure the capacity of an edible substance to produce heat and pain.

That's not too hot for thrill-seeking enthusiasts who are creating new hybridized peppers and chemically extracted chilli products, some of which soar far beyond the Reaper on the Scoville scale. Invented by American pharmacist Wilbur Scoville in 1912, the Scoville unit helps the food industry achieve chilli sauces with precise levels of heat. For chilli extremists, it helps distinguish the devoted chilli-head from the dilettante.

Scoville's original method - the Scoville organoleptic test - employed a panel of human tasters to determine how many times a substance needed to be diluted in neutral solution until people tasted no heat at all. Under this test, the heat of an extract of the mild jalapeño disappears after being diluted about 5,000 times, yielding a Scoville rating of roughly 5,000 , although individual chillies fluctuate between 3,500 and 8,000 . The hotter bird's eye scores between 50,000 and 100,000.

Today, the multi-billion-dollar hot sauce industry measures Scoville ratings with a faster and more objective scientific method based on high-pressure liquid chromotography of pulverized chilli samples in solution. This method detects the concentration of capsaicin, the chemical responsible for chilli heat. Chomp down on a Carolina Reaper and your tongue burns because capsaicin a phytoalkaloid produced by chilli

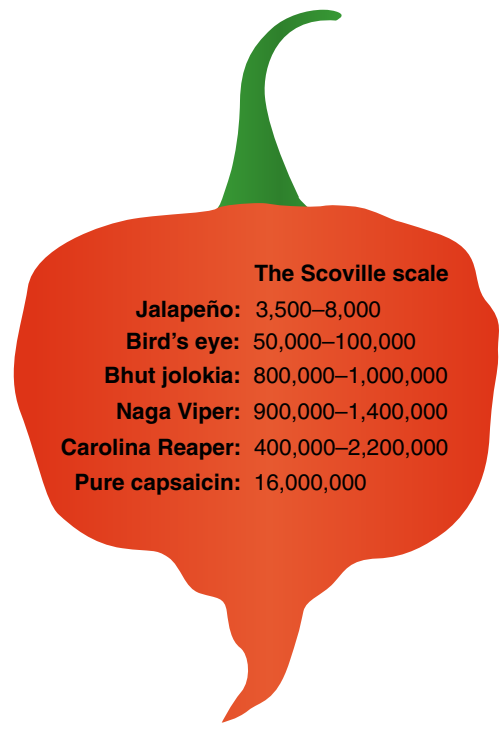

plants - binds to a key temperature receptor, TRPV1, present on nerve fibres. The result is an urgent message sent to the brain - "You're on fire!"

This mechanism reflects an evolutionary adaptation by chilli plants, which spread their seeds more effectively if birds, rather than mammals, eat their fruit. Mammals chew food with teeth, destroying ingested seeds. In contrast, seeds pass easily through the digestive tract of birds, emerging intact to be scattered far and wide. The burn of capsaicin makes most level-headed mammals avoid chilli pepper plants, helping those plants thrive.

The exception is one species of mammal, Homo sapiens, which seems hell-bent on seeking the most intense chilli pain possible. The creator of the Carolina Reaper has recently claimed to have bred a new chilli, named Pepper X, with a Scoville rating over 3 million, well beyond the Reaper. Pepper X awaits confirmation as the world's new hottest chilli by the Guinness Book of Records. But those seeking insane levels of capsaicin agony aren't waiting for selective breeding, and have used chemistry to take chilli extracts into new territory.
The recipe for an extract is simple: immerse pulverized chilli in very pure alcohol, and let the alcohol draw capsaicincontaining oils into the solution. Later, evaporate off the alcohol. I have an extract in my kitchen - a nearly black oil with frightening tinges of exotic orange, which measures around 1.2 million on the Scoville scale. This is useful for ramping up the heat of any salsa. But other extracts are just a click away: Psycho Serum is far hotter than any natural chilli, scoring 6.4 million on the Scoville scale. Or there's Mad Dog 37 Plutonium coming in at 9 million Scoville units, or the elegantly named Death Cap at a ludicrous 13 million, close to the theoretical maximum of 16 million for capsaicin-based extracts.

That number is attained only by a pure crystalline extract of capsaicin - obviously too dangerous to be ingested by any human being. Well, not really, as you can see in a delightful video (https://go.nature. com/2DK7T79) self-recorded by chilli extremist Darth Naga nearly a decade ago. Forty-five seconds after swallowing some pure capsaicin extract, in agony and suffering from tremors and hiccups, Darth bravely manages to put his experience into words: "The heat is unfathomable!" before shutting off the video. Darth survived, fortunately.

For now, 16 million Scoville units is the upper limit to the chilli experience - there's nothing hotter, at least with the biomolecule capsaicin. But other biomolecules carry more punch than capsaicin. Resiniferatoxin, a chemical produced by the cactus-like plant Euphorbia resinifera, is the hottest chemical currently known, 1,000 times hotter even than capsaicin. A pure extract of this stuff scores 16 billion Scoville units. Eating just a gram or two would cause serious internal burns, and possibly death.

\section{Mark Buchanan}

Science writer, based in Europe.

e-mail:buchanan.mark@gmail.com

Published online: 8 January 2020

https://doi.org/10.1038/s41567-019-0766-3

\begin{tabular}{|c|c|c|c|c|c|c|c|c|c|c|c|c|c|c|c|c|}
\hline $\mathrm{m}$ & $e$ & $\AA$ & $\mathrm{S}$ & $\mathrm{u}$ & $R$ & $E_{\mathrm{h}}$ & $F$ & $\Omega$ & ${ }^{\circ} \mathrm{R}$ & $\mu_{0}$ & $\varepsilon_{0}$ & $\alpha$ & $\sigma$ & $\mathrm{V}$ & $\mathrm{R}$ & e \\
\hline
\end{tabular}

